\title{
A Network Approach to Link Visibility and Urban Activity Location
}

\author{
Asya Natapov ${ }^{1}$ (D) Daniel Czamanski ${ }^{2}$. \\ Dafna Fisher-Gewirtzman ${ }^{3}$
}

Published online: 7 July 2018

(C) The Author(s) 2018

\begin{abstract}
Performance of a range of urban amenities is influenced by their accessibility to pedestrians. Success in attracting pedestrians to a particular location depends on how they project visuospatial information. In this paper, we propose an original method for analysing the visuospatial integration of particular locations within a street network. As a case study we analyse the distribution of one type of urban amenities - food and drink public facilities. We represent them in a form of visibility graph as objects of navigational decisions within the street network. To explore how urban facilities, streets and pedestrian visual cognition are interrelated, we create and compare three cases: a street network visibility graph and two visibility graphs of amenities. The first graph is based on the existing, "natural" distribution, while the second is an "artificial", fabricated version of the environment, where urban locations are redistributed evenly across the case study. We study the graphs' global network properties by the use of small-world, and scale-free models. Our results demonstrate that views available for an urban traveller in the existing, "natural" setting had a particular structure. It is built of numerous weakly connected locations coexisting with a small number of hubs with an exceptionally large number of visual connections. Such organisation of urban visibility shows that visuospatial network shares morphological similarities with other natural networks, suggesting that common organizational principles underlie network structure.
\end{abstract}

Electronic supplementary material The online version of this article (https://doi.org/10.1007/s11067-0189411-4) contains supplementary material, which is available to authorized users.

Asya Natapov

a.natapov@ucl.ac.uk

1 Centre for Advanced Spatial Analysis, UCL, London, UK

2 School of Management, Western Galilee College, Acre, Israel

3 Faculty of Architecture and Town Planning. Technion-IIT, Haifa, Israel 
Keywords Urban planning and design - Activity location - Visibility - Spatial cognition · Pedestrians $\cdot$ Third place $\cdot$ Spatial networks $\cdot$ Small-world $\cdot$ Scale-free

\section{Introduction: Walking, Activity Locations and Visibility}

As a consequence of intensified urbanization, urban planners and designers seek to identify the environmental qualities related to the walkability of our future cities. Nurturing urban forms that encourage walking has become an important goal in planning environmentally, socially, and economically sustainable urban areas. Walkability in cities varies, depending on the specific morphology of street network, distribution of urban locations and the availability of visual information.

In urban studies, relationships between these spatial components are systematized by use of mathematical graphs (Ducruet and Beauguitte 2014; Barthelemy 2011). The first and the most accepted of the graph representation methods is the so-called primary graph. This method represents junctions in street networks as nodes, and the street segments that connect junctions as edges (Eichler et al. 2013; Strano et al. 2012 and Barthelemy 2011).

Unlike the simple notation of street junctions, graph methods based on visibility account for the "cognitive complexity" involved in urban navigation. In recent years, there has been a strong degree of growing overlap between graph-based models and the research of human spatial and visual cognition and navigation (Natapov and FisherGewirtzman 2016; Franz et al. 2005; Hölscher et al. 2006). Visual properties are embedded in both the urban environment and pedestrian behaviour, and most pedestrian behaviours are guided by the processing of visuospatial information (Wiener et al. 2009; Golledge and Gärling 2002; Gibson 1950). Visibility is an important determinant of urban vitality; visuospatial characteristics play a central role in navigation performance (Wang et al. 2014 for a review). Especially, such tasks as wayfinding, search and exploration in an unfamiliar urban environment are based on the human sense of sight as a generative feature for shaping spatial memory and behaviour (Wiener et al. 2009).

Weisman (1981) describes taxonomy of features for navigation, which include (a) visual access between key locations; (b) spatial differentiation and (c) layout complexity. Two of these features, visual access and layout complexity, can be quantified by visibility graphs.

A graph representation called axial line graph, represents a network of movement options by showing straight street segments drawn over every longest line of sight as nodes and their intersections as links (Hillier and Hanson 1984). Visibility graph (VG) represents space by imposing a regular or irregular grid on top of it (O'Sullivan and Turner 2001; Krüger 1979). Characteristic point graph implements an alternative VG model that makes use of the human navigational principles in the city; it is built of a set of navigational decision points, street intersections, as nodes in the graph (Jiang and Claramunt 2002).

However, most of the existing graph approaches consider only street networks and ignore functional aspects of the built form, whereas urban uses and activities are extremely important features of the city landscape. They serve as origin and destination points for traveller routes, and are accommodative centres of urban life. Analysing street network connectivity alone without considering functional locations cannot 
present the whole picture of urban realm (Sevtsuk and Mekonne 2012). For instance, axial lines fail to differentiate between the centrality values of a particular location at the different ends of the street segment. All the locations along a street corresponding to one sight line acquire identical centrality.

In this paper we explore how urban uses and activities, streets and pedestrian visual perception are interrelated. What are the organizational principles behind existing urban structure? We seek to define the role that visuospatial properties play in emergence of urban activity patterns; the logic of this phenomenon, one arising from the interaction of urban components such as street networks, land uses, and pedestrian visuospatial cognition.

The emergence of urban uses and activities in cities is inordinately complex process influenced by diverse zoning laws, de jure segregation and more (Loewenstein 1963). However, on the fine-grained resolution, the exact location of new openings in buildings is a spontaneous, bottom-up urban phenomenon, not prescribed by planning authorities. With that, it's known that some of the land uses, such as retail and entertainment places tend to be distributed in a particular form, clustering together (For instance, Fig. 1a). The majority of existing studies focus on the economic principles underpinning this clustering, ignoring the influence of urban morphology and associated human behaviour (Huang and Levinson 2011; Ottaviano et al. 1998). This study seeks to expand the investigation of urban uses and activities distribution, by incorporating the capabilities of human visual perception. Using a graph method that links urban form with the main cognitive property of pedestrians - their visual ability, we link morphology and cognition at the level of streets, buildings, and building entrances, where pedestrian movement becomes central to understanding urban dynamics.

To achieve these objectives, we analysed three cases, based on one origin, the historical centre of Tel Aviv-Yafo, Israel. The first is the street network of the case study. Streets tend to persist in time much longer than other urban elements like

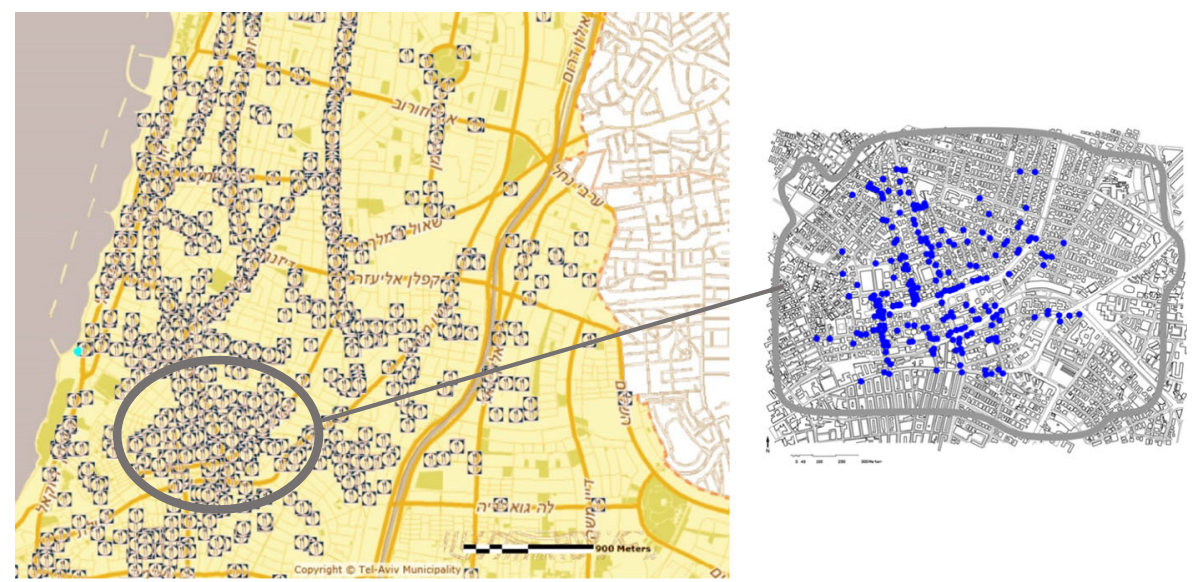

(a)

(b)

Fig. 1 Food and drink facility patterns. (a) The whole city of Tel Aviv-Yafo. (Courtesy of Tel Aviv-Yafo Municipality GIS website); (b) a detailed map of the case study area 
buildings or building uses, and provide a structural basis for further urban development (Vernesz-Mouton, 1989). So, it is important to assess this case in front of the existing knowledge regarding behaviour of the street networks. The second case is an existing, natural distribution of one particular category of urban uses; and the third, is a hypothetical, artificial situation, where the locations of urban uses are systematically modified. Here, urban uses are uniformly allocated according to the existing configuration of streets and buildings.

To compare these cases, we used two models from the network theory: small-world and scale-free. The scale-free model explores the size distribution of the links in the networks, while the small-world model examines the global organization and connectedness of the graph. Small-world and scale-free properties allow classifying different networks in terms of their robustness, efficiency and heterogeneity (Barthelemy 2011; Newman 2010).

We compared these cases in order to understand the impact of existing, natural visibility, and to assess the potential link of urban morphology, urban planning and design. Comparing natural and artificial urban geographies, we evaluated the hypothesis that human visual cognition is a relevant actor in processes of urban evolution. Our expectation was that the small-world and scale-free models would reveal whether these cases were morphologically identifiable, and would thus provide a clearer interpretation of interaction between behavioural and physical qualities in cities.

The rest of the paper is structured as follows: the next section presents existing knowledge about small-world and scale-free phenomena alongside the case study and a new VG representation. Section 3 presents the results of the networks' construction and analysis. Section 4 concludes the paper with an interpretation of the models, implication for urban planning, and suggestions for future research.

\section{Background and Research Methods}

\subsection{Data and Study Area}

We study location patterns of one specific group of pedestrian navigational targets: cafés, restaurants, and other food and drink public facilities. These places have a special meaning in modern cities. Oldenburg (1989) developed the concept of the 'third place', related to these informal public congregation spaces. The concept defines specific social surroundings, distinct from the two usual social environments of home and workplace.

In this research, we used cafés and restaurants located in the historical district of the city of Tel Aviv-Yafo, Israel, called Lev Ha'Ir (Heart of the City), (Fig. 1b). Figure 1a shows the overall spatial patterns of 'third places' in the city. The dataset was collected in the manual survey of the area (Natapov et al. 2013) and includes various kinds of food and drink public facilities. The main criterion for their selection was availability of street seating places. This criterion ensures that the facilities behave as 'third places', i.e. places for urban social interactions. The moderate Israeli climate, and the streetoriented frontages of historical buildings make the district an ideal location for all kinds of 'third places'. The profitability of these places is a function of visit frequency, since they are amongst the most probable navigation targets for tourists and local pedestrians strolling through the area. 


\subsection{Integrative Visibility Graph}

Integrative visibility graph (IVG) used in this study encodes street network and building uses in one unified framework (Natapov et al. 2013), (Fig. 2). The first type of nodes in the IVG consists of street network decision points. These are locations where people make a navigational choice concerning where to head next: street junctions and turning points. The second type of nodes is locations of urban uses and activities. Thus, the graph illustrates a hypothetical visual trajectory of a person looking for a particular activity in the city.

Following the algorithm developed in Natapov et al. (2013), the visibility matrix was built in MathWorks, MATLAB. Using two-dimensional maps of the streets and buildings, the matrix links between the road junctions (centre of the yellow dot in Fig. 2b) and activity locations (centre of the blue dot in Fig. 2b) if they don't intersect with building contours. The visibility is mutual, i.e. the resulted graphs are undirected. Based on this matrix, we constructed two graphs: street network visibility graph (SNVG) and integrative visibility graph (IVG). SNVG summaries all the visual connections between street intersections, while IVG shows connections between both types of navigational points, i.e. intersections and 'third places'. When the graphs are constructed, we used Gephi Network Analysis package (Jacomy et al. 2009), for examination of small-world and scale-free models (for the applied measures see section 2.3).

In similarity to other graph techniques, IVG represents urban environment in a very abstracted way. Real-world settings consist of the much wider range of psychical properties of viewers and environments. These include size, contrast, colour, shape, texture, pattern, and complexity of the viewed elements, their static or dynamic position, atmospheric conditions, distance, angle of observation, etc. (Wang et al. 2014; GretRegamy et al. 2007; Shang and Bishop 2000). However, according to Bishop (2002) in areas with transparent skies, visibility modelling out to $20 \mathrm{~km}-30 \mathrm{~km}$ is justified. In our case, when the method is applied to the dense urban conditions, pure effect of the line of sight is the most influential, and other factors could be neglected.

This study focuses on one specific sector of uses and activities ('third places'), however the graph is suitable for other retail street-fronting urban facilities. The

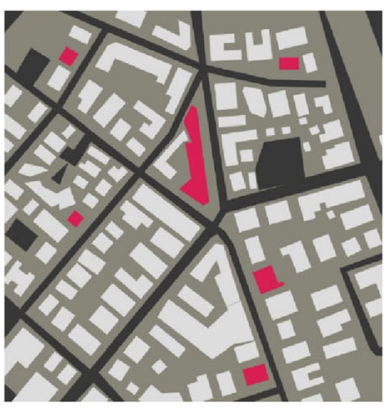

(a)

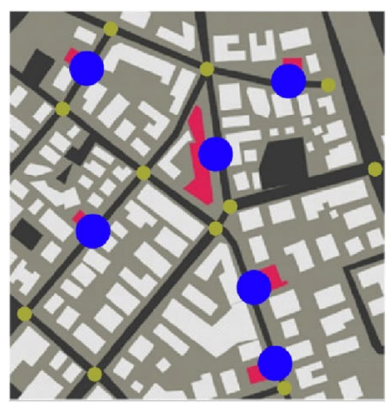

(b)

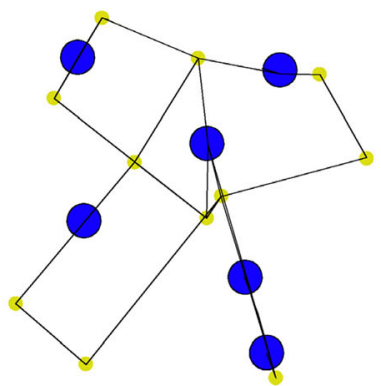

(c)

Fig. 2 IVG construction: (a) urban layout; roads are shown in black, open space in grey, buildings in light grey, and building with activities in red; (b) two types of nodes, where navigational decisions are taken. Yellow nodes are street intersections and blue nodes are activity locations; (c) nodes are connected by visibility lines as edges in the graph 
possibility of breaking a graph into two separated graphs, SNVG and IVG, allow us to use both street networks and building uses as units of the analysis, and to compute network measures separately for each of them. Therefore, we are able to account for a particular location in the city, such as building use, throughout the visibility network. Neither of these is addressed in most urban graph methods.

\subsection{The Small-World and the Scale-Free Models}

In this paper, we apply two models that examine global properties and overall organization of the networks - a small-world model and a scale-free model. A small-world network is a type of graph in which most nodes are not located closely to each other, but at the same time can be reached by means of a small number of intermediate steps. This model examines an overall behaviour of the system and not the individual status of a particular node as, for example, in the centrality model - the model indicating the most important nodes within a graph (Newman 2010).

Small-world graphs are classified according to two independent structural features, namely the average shortest path length and the clustering coefficient (Watts and Strogatz 1998). The average path length in the graph $G$ with $N$ vertices is given by:

$$
L=\frac{1}{N(N-1)} \sum_{j \neq i} d\left(v_{i}, v_{j}\right)
$$

where $d\left(v_{i}, v_{j}\right)$ is the length of the shortest path between the vertices $v_{i}$ and $v_{j}$.

The clustering coefficient is a measure of the extent to which the neighbours of a vertex $v_{i}$ are linked to each other. It measures the proportion of actual connections between the neighbours of a node divided by the number of all possible connections. The clustering coefficient of the vertex $v_{i}$ is defined by:

$$
C_{i}=\frac{2 l_{i}}{m(m-1)}
$$

where $l_{i}$ is the number of edges among the immediate neighbours of the vertex $v_{i}$ and $m$ the number of immediate neighbours of the vertex $v_{i}$. Finally, the clustering coefficient $C$ of a graph $G$ is defined as the average of the vertex clustering coefficients. Whether a network is a small-world is determined by comparing the value of $L$ and $C$ with those obtained for the randomized version of the network, i.e. for a network with the same $N$ and in which the edges are randomly distributed, with a uniform probability, among all the nodes. In a small-world network, $L \approx L_{\text {random }}$ and $C \gg C_{\text {random }}$ and on the other hand $L \ll L_{\text {regular }}$ and $C \approx C_{\text {regular }}$.

In regard to street networks, we inquire whether our way of representing streets, SNVG, confirms other small-world oriented studies. These studies have widely diverging results, depending on the representation procedures. As has already been mentioned, street networks can be converted into graphs using different methods of representations, such as nodes and edges corresponding to individual streets, street intersections, longest sights, street names or angularity. While traditional, geometric, road-based models fail to observe small-world properties, a new street-based modelling 
approach has obtained opposite results, by observing small-world properties for the diverse methods of graph representations, e.g., (Jiang et al. 2014; Jiang 2007; Porta et al. 2006; Jiang and Claramunt 2004).

Another model examined in this study is the so-called scale-free network, i.e. a network whose degree distribution follows a power law. The degree centrality is defined as the number of links a node has. In our case the degree centrality measures how many locations can be seen from each other location within the given morphological conditions.

In general, scaling (the study of distribution and the scale invariance) has a long history in urban discipline. The evolution of social and economic life in cities increases with population size: wages, income, gross domestic product and bank deposits scale super linearly, over different years and nations, with statistically consistent exponents (Bettencourt et al. 2007; Andersson et al. 2006; Florida 2004; Barabasi and Albert 1999). Since the early study of random graphs performed by Erdös and Renyi (1959), the degree distribution

$$
P(k)=\operatorname{Pr}\left[\operatorname{deg}\left(v_{i}\right)=k\right]
$$

has become a common way of classifying graphs into categories. In random graphs $P(k)$ asymptotically follows a Poisson distribution. Poisson distribution expresses the probability in fixed intervals with a known average rate, while an exponential distribution describes relationships in which a constant change in one quantity gives the same proportional change, occurring as an exponent in the other quantity (Barthelemy 2011). In the scale-free graphs, degree distribution follows a power law (Barthelemy 2011; Clauset et al. 2009; Bettencourt et al. 2007):

$$
P(k) \cong \frac{1}{k^{\gamma}}, \gamma>1
$$

Power law function describes a relationship between two quantities, where a relative change in one quantity results in a proportionate, relative change in the other quantity: one quantity varies as a power of another. It is called scale-free because zooming in of any part of the distribution will not change its shape. There are a few, but significant, number of nodes with a lot of connections, and a trailing tail of nodes with very few connections at each level of magnification. It means that in a scale-free network, the structure and dynamics are independent of the network size $N$.

SNVG, examined in section 3.3 is a visibility graph of the street network. With regard to other visibility graphs, Jiang (2006) showed that topographic surfaces exhibit an exponential distribution rather than a power law, i.e. it is not scale-free, in contrast to many other real world networks. In studies of street networks, various results of degree statistics can be found. The comparative investigations in (Cardillo et al. 2006 and Porta et al. 2006) reveal scale-free degree distributions for the vertices of spatial graphs if the ICN (intersection continuity negotiation) principle is implemented. Carvalho and Penn (2010) studied the distribution of the length of open space linear segments, and 
showed that their cumulative distribution displays power-law tails. However, it has been reported by Jiang and Claramunt (2004) that, under the street name approach, the street graphs exhibit small-world characteristics but scale-free degree statistics can hardly be recognized. In general, street networks do not provide sufficient data to draw conclusions about the universality of degree statistics.

\section{Network Construction and Analysis}

\subsection{Comparing two Urban Situations: 'Natural' and 'Artificial'}

In this section, we present a real-world, natural, and a modified, artificial, distribution of 'third places' in the case study. The initial step of the graph construction was to map the decision points within the street network, i.e. points where a traveller would make route decisions (Fig. 3a). Then, different types of drinking and eating street facilities were marked at the entrance to the buildings (217 in total, Fig. 3b).

To investigate the impact of natural, existing visibility, which emerged on the site spontaneously, we created a control environment by systematically modifying the locations of 'third places.' We redistributed the same given number of 'third places' in an identical setting (street structure and building arrangement) as uniformly as possible without violating the morphology and spatial logic of the urban environment.

To achieve this uniformity we proceeded methodically as follows. First, we superimposed a grid of equal cells on the existing arrangement of streets and buildings (Fig. 4a). Part of the cells was deactivated following particular boundary areas of the case study. A single 'third place' would be located in each grid cell to ensure the desired uniform distribution. To allow for 217 'third places' as in the original study, 217 active grid cells were thus necessary and the cell size was correspondingly calculated to be $113 \mathrm{~m} / 85 \mathrm{~m}$. Every activity was placed inside the boundaries of its cell manually in accordance with the urban morphology and spatial logic (Fig. 4b). This requires, particularly, that the 'third places' resided in buildings are being projected outside with

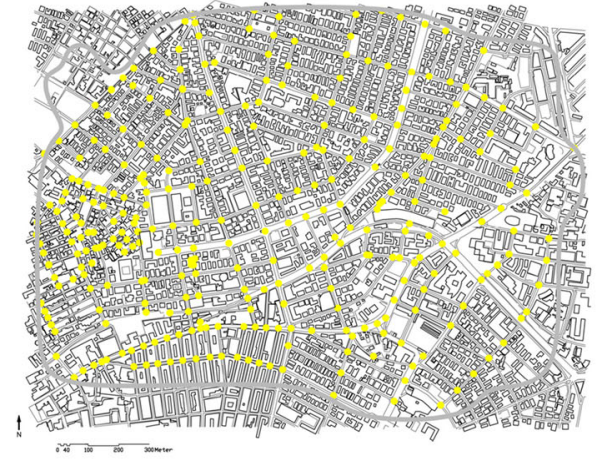

(a)

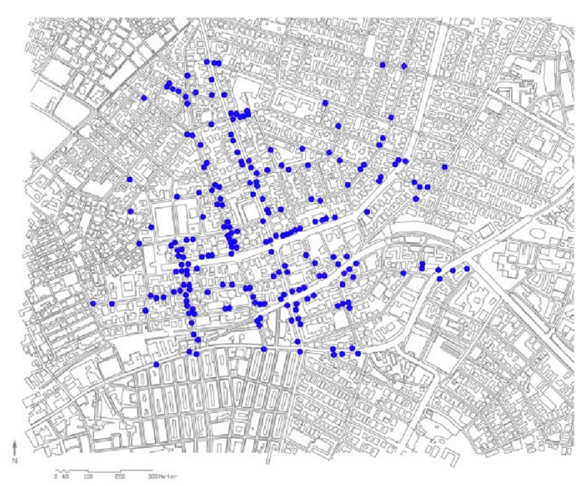

(b)

Fig. 3 (a) Points of taking decision within the street network - junctions and turning points. (b) 'third place' decision points - food and drink public facilities 


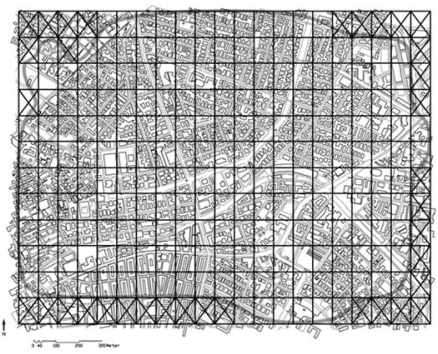

(a)

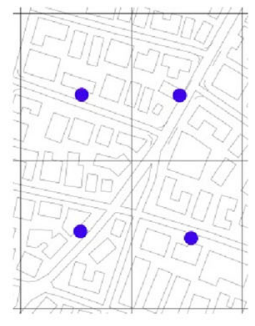

(b)

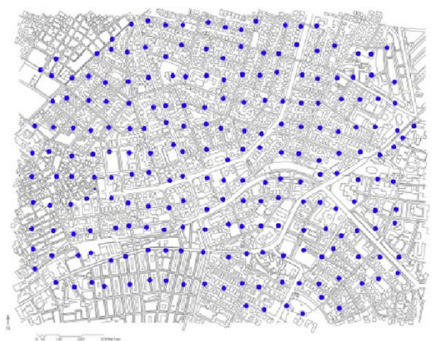

(c)

Fig. 4 IVG modification procedure: (a) a grid imposed on the case study. (b) zoom in to the several grid cells with a new allocation of the 'third places'. (c) redistribution of the 'third places' in the area according to the grid

pavement seatings that face streets, and that they make use of the open space between surrounding buildings, as would be the case in the real world. In consequence, we developed an artificial urban situation with the 'third places' distributed evenly in our area of interest (Fig. 4c).

In applying our analyses both to the original network of activities and to the layout resulting from the procedure described above, we created a comparison that enabled us to assess potential links between visibility and urban morphology in a weighed and controlled manner.

Thus we derived three visibility graphs from the two environments, the original and the artificially uniform. Each graph is visualized in Fig. 5 in a geo-coordinated and a non-geo-referenced views. Figures $5 \mathrm{a}$ and $\mathrm{b}$ show an SNVG graph. Its nodes are decision points embedded in the street network, and the edges indicate their intervisibility. The second graph (Figs. $5 \mathrm{c}$ and d) is an IVG of the original, natural network. Its set of nodes represents street network decision points (shown in yellow) combined with activity locations (eating and gathering places, shown in blue). The following visualization (Figs. 5e and $\mathrm{f}$ ) is a corresponding IVG representation of the artificial environment with the rearrangement of the 'third places.'

All the graphs are undirected, i.e. the visibility is mutual. Non-geo-referenced layouts in Fig. 5 are created by the algorithm that pull together all the linked nodes and pushed apart non-linked. In such visualization, the central hubs of the graphs are clearly recognizable. These hubs identify urban locations with high visibility.

Details of the constructed graphs are shown in Table 1 (relevant information explaining graphs' details is found in Appendix 2).

\subsection{Small-World Model}

The values of average path length $L$ and the clustering coefficient $C$ are computed respectively against those of the identical size regular and random graphs of SNVG, natural IVG and artificial IVG. The random graphs are generated in the network analysis package, Gephi (Jacomy et al. 2009), using the probability of links distribution equal to:

$$
p_{i}=\frac{2 E}{N(N-1)}
$$


The calculations of $L$ and $C$ in actual and random graphs were performed in the same package and for the regular graphs it is straightforward following Jiang (2005) (see supporting files).

$$
\begin{gathered}
L_{\text {regular }} \approx \frac{N}{2 M} \\
C_{\text {regular }}=\frac{3}{4} * \frac{M-2}{M-1}
\end{gathered}
$$

The networks were tested for small-world properties using the S-statistic suggested in Humphries and Gurney (2008). They define $S$ :

$$
\begin{gathered}
S=\frac{\gamma_{g}}{\lambda_{g}} \\
\text { where } \gamma_{g}=\frac{C_{g}}{C_{\text {rand }}} \text { and } \lambda_{g}=\frac{L_{g}}{L_{\text {rand }}}
\end{gathered}
$$

A network is considered to be a small-world if $S>1$.

$\boldsymbol{N}$ - number of nodes, $\boldsymbol{E}$ - number of edges, $\boldsymbol{M}$ - average degree, $\boldsymbol{L}$ - average path length, $\boldsymbol{C}$ - clustering coefficient, $\boldsymbol{S}$ - S-statistic according to (8) and (9).

The results demonstrate that all three graphs exhibit small-world properties (Table 2). All S-statistics are higher than 1. Also the average path length of the graphs is higher than that in the corresponding random graph, and significantly lower than in the corresponding regular graph. At the same time, in each case the clustering coefficient is close to that of the corresponding regular graph, so the graphs are highly connected.

\subsection{Scale-Free Model}

In this section we examine and compare a degree distribution of the three visibility graphs by several tests (Fig. 6 and Table 3). The first test approximates these distributions by exponential and power law curves using a standard least-square fitting method (named LS in Table 3). The second test follows a novel technique by Clauset, Shalizi and Newman (2009), (named CSN in Table 3), who proposed an advanced statistical framework for quantifying power-law behaviour in empirical data. This approach combines maximum-likelihood fitting with goodness-of-fit tests based on the Kolmogorov-Smirnov statistic and likelihood ratios. The method allows to find the threshold (xMin) and the scaling parameter Alpha such that the distribution most likely to be scale-free above the xMin with the parameter Alpha. In other words, they propose to look for the longest tail which is most likely scale-free in each distribution. Clauset et al. (2009) found that in practice few empirical phenomena obey power laws for all values of $x$, because of the sensitivity to noise or fluctuations in the tail of the distribution. More often the power law applies only for values greater than some minimum xMin. Therefore, it is suggested to examine the tail where large fluctuations occur - the part of the distribution representing large but rare events. 


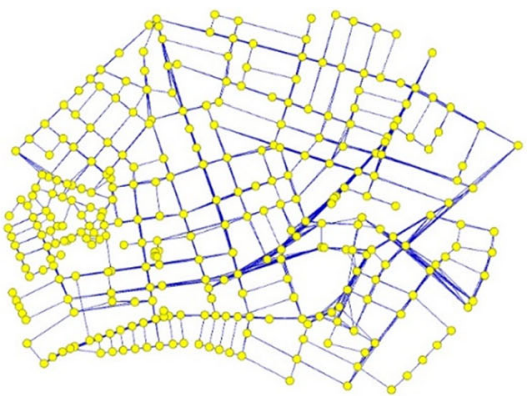

(a)

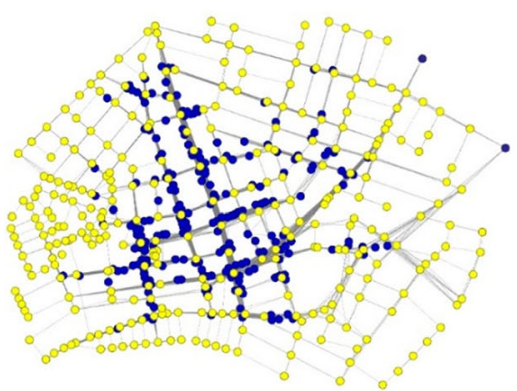

(c)

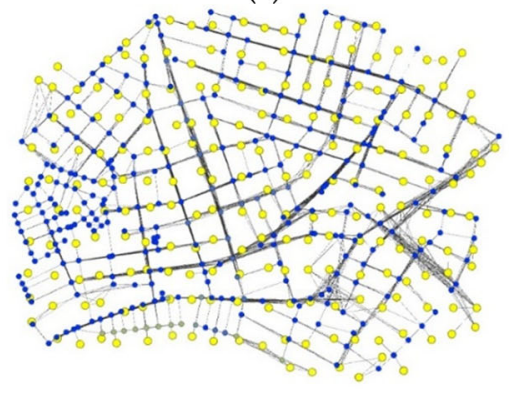

(e)

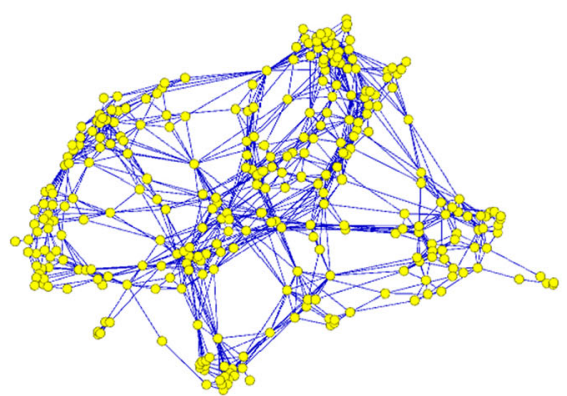

(b)

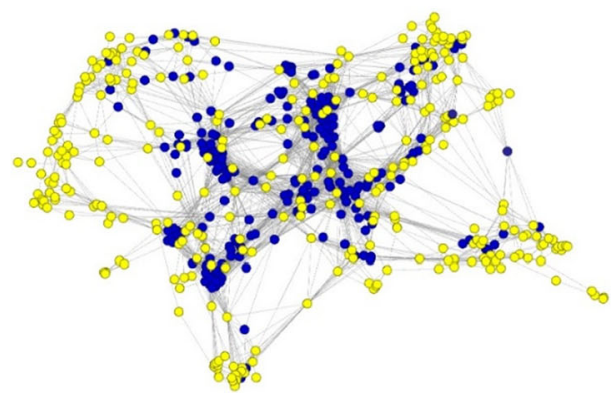

(d)

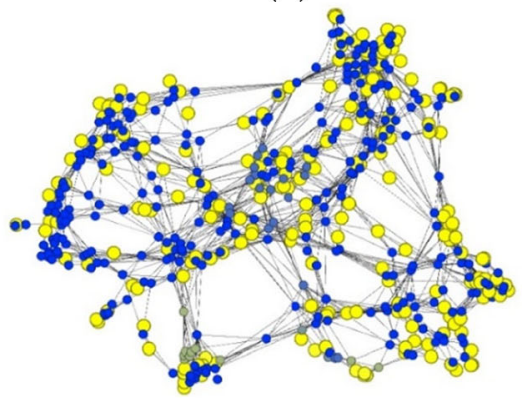

(f)

Fig. 5 Visibility graphs built of street intersections (shown in yellow), and 'third places' (shown in blue). (a) SNVG geo-coordinated layout; (b) SNVG non-geo-referenced layout; (c) natural IVG geo-coordinated layout; (d) natural IVG non-geo-referenced layout; (e) artificial geo-coordinated IVG; (f) non-geo-referenced artificial IVG

In the first test we exclude the nodes with the smallest degrees 1 and 2 (thus setting effective $\mathrm{xMin}=3$ in the CSN terminology). These nodes are located on the dead-end streets, or on the margins of the case study area (Appendix 1 for details). R squared values of SNVG and artificial IVG show that these graphs exhibit an exponential distribution rather than a power law distribution, i.e. it is not scale-free. Unlike them, the natural IVG degree distribution seems to be more power law-like. In fact, the shorter the tail of the distribution we take with the LS method, the better it fits a power law distribution.

Following the Clauset et al. (2009) method we conduct several experiments using software they suggested (plfit.m and plpva.m). We first try to fit all of the cumulative 
Table 1 Details of three visibility graphs

\begin{tabular}{lll}
\hline & \# of nodes & \# of edges \\
\hline Street Network Visibility Graph & 309 & 1338 \\
Natural IVG & 526 & 4172 \\
Artificial IVG & 526 & 5000 \\
\hline
\end{tabular}

distribution, using plfit.m suggested by the authors, within the acceptable range of alphas - between 1.5 and 3.5, and then with the larger parameters as well. No one of the distribution is successfully fitted with $p$-value $>0.1$. Hence, this method suggests that we should reject the hypothesis that either of our distribution follows power law.

Next, for each distribution we looked for the longest tail where there is a fit with a larger scaling parameter with the significance level $p>0.1$. It appears that approximately last third (n-Tail is the number of nodes in the tail and n-Tail \% is the weight of the tail) of each distribution can be fitted by a power law function with the parameters greater than 4 (Alpha-tail). This shows that all three graphs have long tails that could be considered scale-free. So strictly speaking the CSN method does not show that a likely power law behaviour of the natural IVG is more plausible then the other graphs.

However, using this method we can see that SNVG has relatively heavier tail $(39 \%$ of nodes) that is likely scale-free. Comparing the two versions of IVG, we observe that the natural IVG likely scale-free tail consists of nodes with degrees 18 to 66, while the artificial one has degrees of 12 to 31 . This means that the natural IVG scale-free tail is longer and in these aspects the natural networks stay apart from the artificial one. Despite the fact that we cannot justify the exception of the natural IVG with respect to the other two graphs, we see that the natural IVG distribution fits the best the description "the most locations have approximately the same low level of visibility, while only a few intersections and urban activity locations have extremely high levels of visibility" which is usually designates the scale-free property.

Table 2 Small-world measures of SNVG and the IVGs

\begin{tabular}{|c|c|c|c|c|c|c|}
\hline & $N$ & $E$ & $M$ & $L$ & $C$ & $S$ \\
\hline SNVG & & & & & & 13.14 \\
\hline Actual graph & 309 & 1338 & 8.66 & 4.377 & 0.519 & \\
\hline Regular graph & 309 & 1338 & 8.66 & 17.84 & 0.65 & \\
\hline Random graph & 309 & 1338 & 8.66 & 2.883 & 0.026 & \\
\hline Natural IVG & & & & & & 13.48 \\
\hline Actual graph & 526 & 4172 & 15.86 & 3.74 & 0.59 & \\
\hline Regular graph & 526 & 4172 & 15.86 & 16.58 & 0.70 & \\
\hline Random graph & 526 & 4172 & 15.86 & 2.57 & 0.03 & \\
\hline Artificial IVG & & & & & & 10.71 \\
\hline Actual graph & 526 & 5000 & 9.52 & 4.62 & 0.61 & \\
\hline Regular graph & 526 & 5000 & 9.52 & 27.62 & 0.66 & \\
\hline Random graph & 526 & 5000 & 9.52 & 2.44 & 0.03 & \\
\hline
\end{tabular}


SNVG
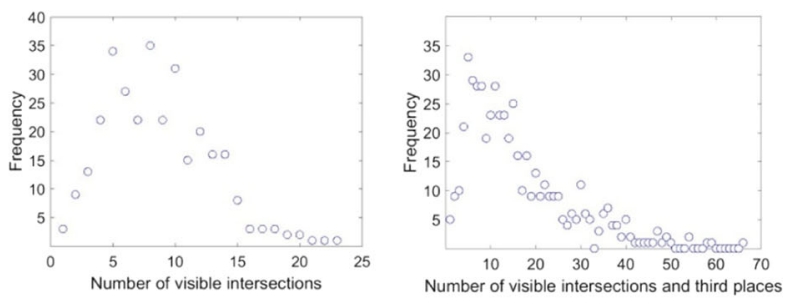

(a)
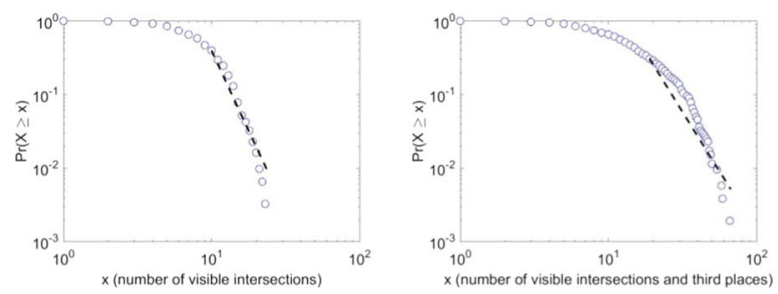

Natural IVG

Artificial IVG

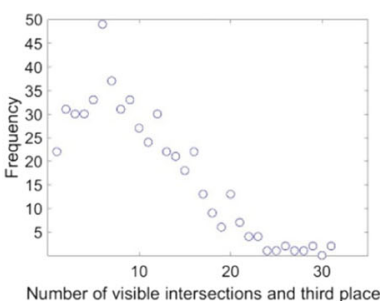

Number of visible intersections and third place

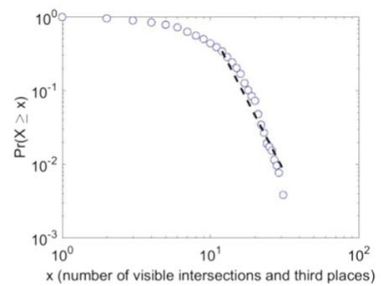

(b)

Fig. 6 Degree distribution of the three studied graphs: SNVG, natural IVG and artificial IVG. (a) Degree frequencies in the three graphs; (b) cumulative distribution functions and the power law fit of the three networks on the loglog scale

To understand deeper the differences between characteristics of visibility in natural and artificial situations, we conducted an additional test examining a superposition of the street network and 'third place' locations. As street network remains the same in both cases, here we compared both IVGs on the basis of the existing street network (Fig. 7). In both Figs $7 \mathrm{a}$ and $\mathrm{b}$, street network visibility lines are shown in blue

Table 3 Correlation of the studied graphs to the distribution functions

\begin{tabular}{llll}
\hline & SNVG & Natural IVG & Artificial IVG \\
\hline $\begin{array}{llll}\text { Nodes } \\
\text { xMax }\end{array}$ & 309 & 526 & 526 \\
Exponential LS & 23 & 66 & 31 \\
$\quad \mathrm{R}^{2}$ & & & \\
Power law LS & 0.81 & 0.88 & 0.84 \\
$\quad$ xMin-LS & 3 & 3 & 3 \\
Alpha-LS & 1.69 & 1.49 & 1.49 \\
$\mathrm{R}^{2}$ & 0.6 & 0.81 & 0.67 \\
Power law CSN & & & 18 \\
$\quad \mathrm{xMin}$ & 10 & 12 & 4.81 \\
Alpha-tail & 5.18 & 4.06 & 179 \\
n-Tail & 122 & 177 & $34 \%$ \\
n-Tail\% & $39 \%$ & $34 \%$ & \\
\hline
\end{tabular}


according to the decreasing order of the degree, while the visibility of the activity locations are shown in red. There is a form of high "peaks" exhibited by the existing, natural distribution of the activities. In Fig. 7a we highlight the peaks to show the significant contribution of natural 'third places' in archiving higher degree of overall visibility. In contrast to this, almost all the visibility lines belonging to the artificial distribution are below the streets' visibility, with similar - but much lower - peaks around the street intersections (Fig. 7b). This "peaky" behaviour of the natural "third places' was captured also in the previous test by the scale-free tail consisting of nodes with degrees 18 to 66 , in contrast to the tail of the artificial IVG with degrees of 12 to 31. The artificial IVG has 5000 edges, i.e. available views, while the natural IVG has only 4172. Nevertheless, the artificial, even distribution produces more homogeneous patterns, despite the bigger number of connections.

\section{Summary and Discussion}

\subsection{Discussion of the Findings}

In this study, we examined the visuospatial properties of one predefined urban activity, 'third places' - cafés, restaurants, and other food and drink public facilities distributed within the street network. We sought to find the organizational principles behind the

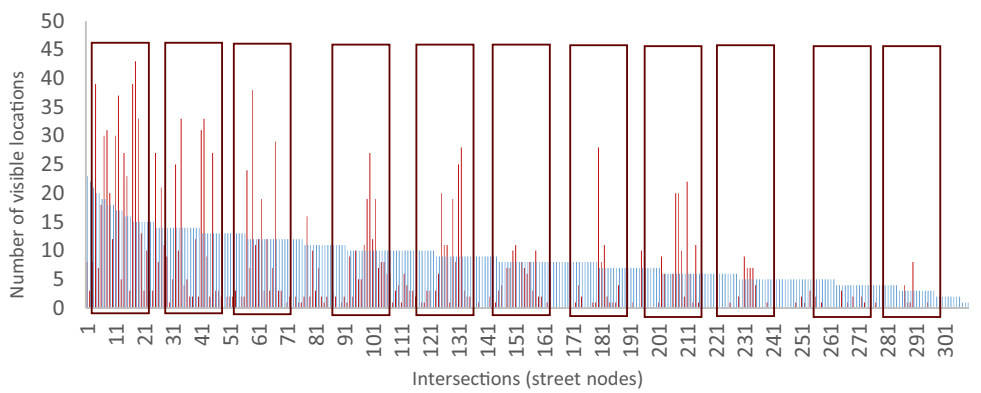

(a)

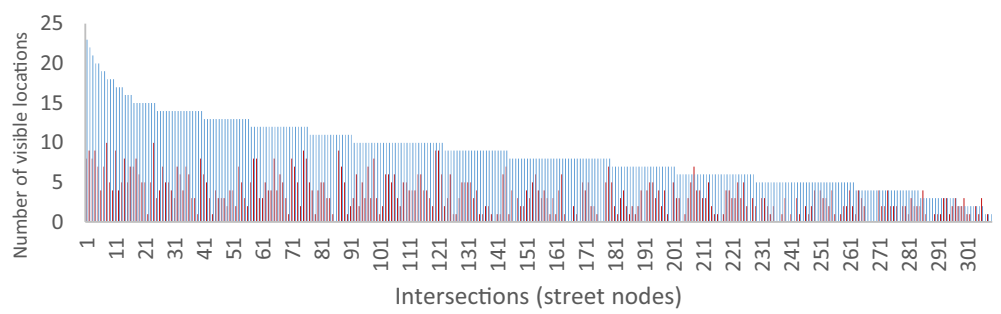

(b)

Fig. 7 Visibility of urban locations in front of the decreasing order of the street network visibility. All street network intersections (shown in blue) are placed in the decreasing order of the degree. In front of them visibility of the corresponded 'third places' is shown in red. In (a) real urban setting exhibits peaks of the functional visibility; in (b) artificial IVG shows that almost all the functional locations are lower than the street intersections 
existing structure emerging from the interaction of urban components, such as street network, building uses, and pedestrian visual cognition.

We represented the urban environment as a chain of pedestrian navigational decisions, in form of a mathematical graph. We created three graphs: a street network visibility graph, and two functional visibility graphs, one existing in reality and other an artificially fabricated graph. The last presented a systematically modified environment, where pedestrian targets, food and drink public facilities, were uniformly distributed within the given street network. Using visual access as a key generative factor in the construction of the graphs, we sought to understand the role played by visual perception in the emergence of urban patterns. We compared the graphs' global network properties as small-world, and scale-free models.

Our results show that all the studied graphs - SNVG, natural IVG and artificial IVG - exhibit small-world properties. That means they are typified by many local interactions, and by a smaller number of inter-area connections. They contain hubs, which have connections between almost any two nodes. This follows from the defining property of a high clustering coefficient; most pairs of nodes are connected by at least one short path. These hubs are highly connected urban locations from where most other strategic locations are easily seen and reachable.

SNVG, discussed in this paper, expands existing knowledge on the behaviour of street networks. Since streets can be converted into graphs using different methods of representations, the results show wide variety. Traditional, road-based method fails to satisfy small-world properties (Sainte-Marieork 2004). But when streets are represented as nodes and edges corresponding to individual streets and street intersections, or by named-based and angularity-based methods, the small-world properties are observed (Jiang et al. 2014; Porta et al. 2006; Jiang and Claramunt 2004). Finding the same pattern on streets as visuospatial, navigational choices we emphasize the impact of visibility on streets "small-worldness."

Natural IVG and its artificial version introduce a new type of urban network, based on the behavioural principals of visual perception. The fact that these networks are small-world opens a new direction in network research. Sharing statistical and morphological similarities with many complex systems - including food chains, metabolite processing networks, networks of brain neurons, voter networks, telephone call graphs and social networks, suggests that common organizational principles might underlie these processes.

Next model, the scale-free, examines distribution of the degree centrality - in our case, the number of views available for a traveller in the city. Methods for detection and characterization of scale-free characteristics are still undergoing changes and development by a network science community. For a long time spatial networks were believed to be scale-free, however recent works have shown that such distributions are much rarer than previously believed (Broido and Clauset 2018). In our examination according to the standard least-square fitting method, only the natural IVG was a scale free, while according to the method suggested by Clauset et al. (2009) all the networks are not scale-free, but their tails are.

Findings on the SNVG that has an exponential distribution rather than a power law, confirms and reinforces the existing knowledge on visibility graphs (Jiang 2005). Several studies also found scale-free degree distributions in street networks, using other graph representations (Cardillo et al. 2006; Carvalho and Penn 2004; Porta et al. 2006). 
However, there is still not enough data to draw comprehensive conclusions about the universality of streets' degree statistics across the various street representation and analysis methods.

Examining behaviour of the 'third places' in the possible scale-free distribution of the natural IVG, we found that the most urban locations have the same low visibility, while only a few cafes and restaurants, have extremely high level of visibility. If we compare the fit of the SNVG to the fit of the IVG (Fig. 6), we can see how the distribution of the visual connections has been changed with addition of the activity locations. Such comparison shows the role played by activity locations in the overall distribution of the natural IVG. Natural IVG, which has fewer connections than the artificial one, produces much more heterogeneous pattern of visibility (Fig. 7). This pattern is considered to be an optimal navigational structure, balancing local and global network efficiency.

\subsection{Interpretation of Network Findings in Real-World Situation}

The tail behaviour of the natural IVG, suggests that the number of views to urban locations is scale-invariant. It means that at various resolutions the morphology yields similar visuospatial results. IVG heterogeneous pattern represents the number of views faced by a traveller in the real environment. This pattern is not homogenous, but has a particular structure with many visually segregated locations, as well as a few locations with a large number of connections.

In Fig. 8 all existing locations of the natural environment are divided into two groups, based on the possible power law behaviour. Group A shows the tail of the degree distribution which is likely scale-free and includes the most visually connected places. This group consists of only $34 \%$ of the locations (177 out of 526), with a degree higher than 18 . While group B includes places with a considerably smaller number of views and comprised of the majority the locations $(66 \%, 349$ out of 526) with a degree less than 18 .

To understand why locations belonging to group A are more attractive than those of group B, we inspected the physical conditions of the two samples from each group. Fig. 9a shows the sample on the map of the case study. Samples from group A have a high degree: The first (A1) of 66 visual connections and the second (A2) of 42, while both samples from group B (B1 and B2) have only 3 connections.

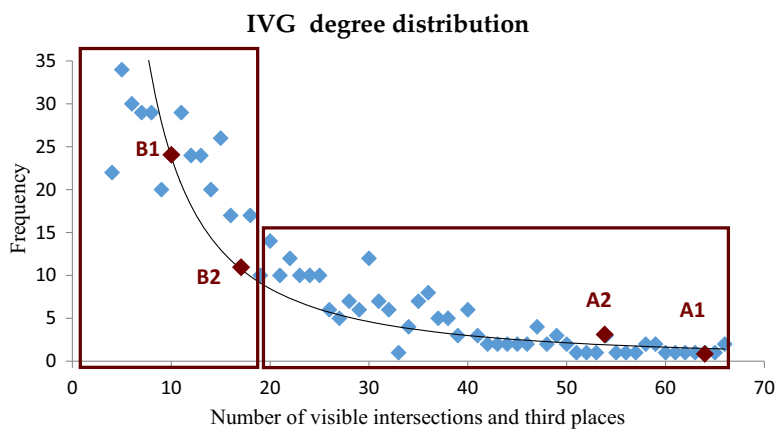

Fig. 8 Approximation of the natural IVG degree distribution with two groups: A-highly connected locations, B-less connected locations 
Highly connected places from group A are located on the intersection of the main road in the area, Allenby Street, and other major streets. In Fig. 1b, which shows all the facilities in the area, it can be seen that there are many other 'third places' located on the same intersections. Less visually connected places from group B, on the contrary, are located not on the intersections, but along quiet, short, secondary streets.

Figs in $9 \mathrm{~b}$ illustrate the real-world physical conditions of the four samples. The reasons for the differences between them can also be found in the width of the streets. It is clear that due to the wide open space between the buildings in Figs A1 and A2, long and distant views are enabled. Space in the less connected locations (B1 and B2) is more controlled and small in size; thus, distant views are blocked.

Highly connected locations, like those in Figs A1 and A2, act as hubs which change the way traveller navigates the network. The more pronounced the hubs are, the more effectively they shrink the distance between urban locations. Therefore, this distribution of urban visibility depicted in the IVG, captures the efficiency of pedestrian visual search.

\subsection{Significance and Further Implementation of the Modelling Technique}

Historically urban street planning and design has been led by traffic engineers who have given priority to vehicles. As the result many streets around the world are unattractive for walking people travelling along the street, or using the street as a destination for different activities. Recently, this attitude has started to change and urban policy makers started considering streets not only as traffic means, but also in the wider urban context, as both as destinations on its own right. Jones et al. (2008) introduced a concept of "Link and Place" to analyse streets on a basis of the needs of people, rather than vehicles. As a Link, a street functions as a movement channel, and as a Place, a street is a destination in its own: a location where activities occur on or adjacent to the street. IVG representation used in our study encodes street network and street activities in one unified framework. Therefore it allows to combine "Link and Place" concepts in one graph-based method and to examine street connectivity together with consideration of functional locations, hence presenting more comprehensive picture of urban realm.

Furthermore, our findings suggest the utility of relating urban dynamics to complex network theory, creating a new link between behavioural and spatial processes in cities. Traditionally complex network theory explains the evolution of scale-free phenomenon by "growth" and "preferential attachment" (Xie and Levinson 2009; Barabasi and Albert 1999). Nodes that existed for longer time in the network had more chances to get connected by newly created nodes. Also, those new nodes were likely to be introduced to the network by hubs. In urban environments, this mechanism means that new activity locations are most likely to be open in places that are visually connected to the hubs (i.e. the most visible intersections), or to the hubs of other activities (i.e. the most visible 'third places').

Typically, the exact locations of cafés and restaurants are not designed by planning authorities, but are the result of bottom-up processes, intrinsic to the way cities grow and evolve. By examining visual integration of food and drink 


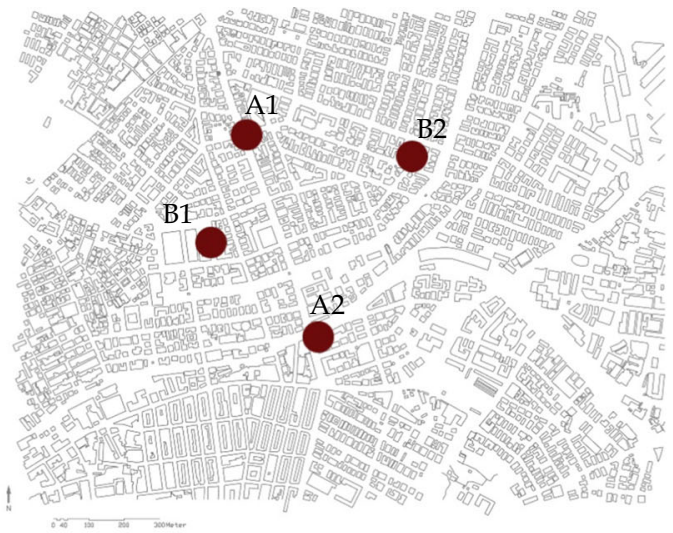

(a)

Samples from Group A

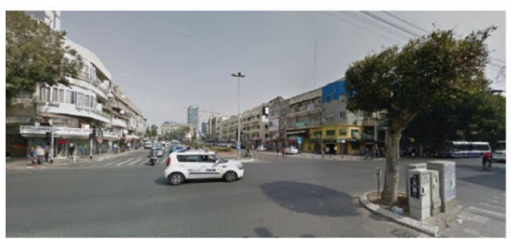

Intersection Yehuda ha-Levi and Allenby Streets

Degree: 66 visual connections

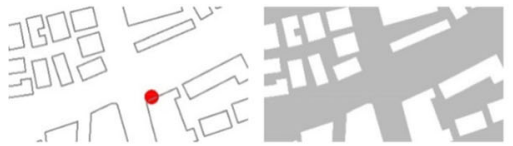

(A1)

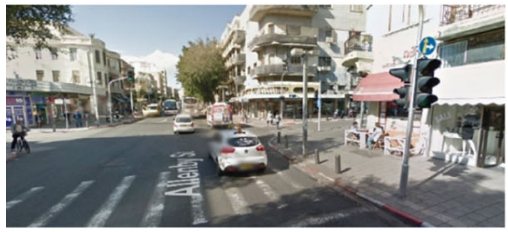

Intersection Maze and Allenby Streets

Degree: 42 visual connections

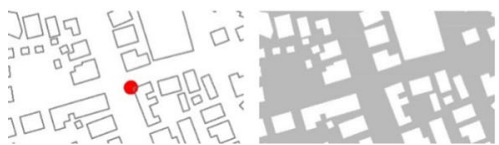

(A2)
Samples from Group B

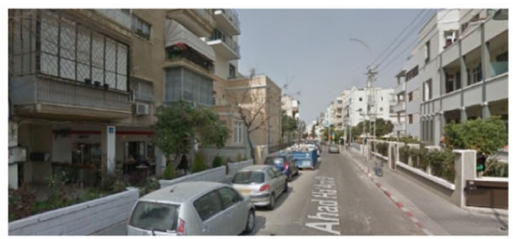

Ahad ha-Am Street

Degree: 3 visual connections

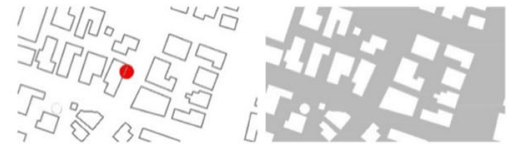

(B1)

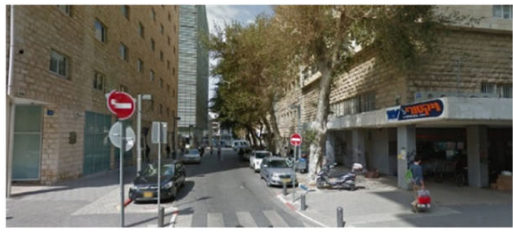

Ahuzat Bayit Street

Degree: 3 visual connections

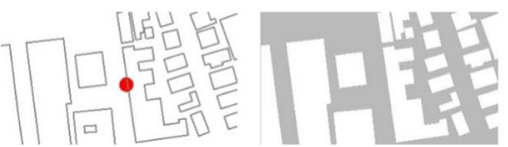

(B2)

(b) 
Fig. 9 Comparison between samples of the highly and weakly connected locations: (a) maps of the case study with the samples; (b) the physical conditions for two cases from group A and group B (Courtesy of Google Maps)

public facilities in the city we provide here an alternative explanation for the processes behind their spatial concentration. Network approach allows us to study the city as a formal emergent phenomenon, with a "hidden hand" guiding its growth, and no top-down decision-maker guiding its physical and spatial organization (Batty 2013; Batty 2003). Therefore, observed network properties have important implications in terms of spatial modelling, and can assist researchers in understanding the mechanisms that underlie the emergence of urban activities. Further it could be used by both research and planning communities in simulations that evaluate wayfinding, pedestrian risks and search efficiency within urban environment. Such simulation models could investigate the interaction between cities's spatial and cognitive features, e.g., how individuals interact with the urban space, and how this space constantly evolves and transforms from the bottom up in response to the behavioural patterns. In addition, this method may be helpful for planners when considering the issuance of zoning and building permits for new buildings that would alter the visuospatial fabric.

In this paper we focused only on one type of urban amenities, however, the method could be expanded for use of planners, designers or developers involved in selecting sites for both public (e.g. parks) and private spaces in cities. Particularly, it could be useful for business owners (e.g. of retail or cafes) to select new sites. It's known that diverse visuospatial attributes, such as viewpoints or vistas, have considerable economic significance and value (Lang and Schaffer 2001).

The study reflects the inherent limitations arising from the representation method, and the case study biases. First, the IVG in its current form is limited to two-dimensional representation that ignores additional factors affecting visual perception. To improve reliability of the method, we propose in the future to integrate a wider range of psychical properties. These properties could include distance, size, colour, shape, texture, complexity of the viewed urban features, and atmospheric conditions. Second, the tests are performed only on the three cases: street network, natural distribution of uses and artificial distribution of uses. We suggest to expand this examination by including additional urban environments with diverse urban morphologies coming from various history of planning.

To summarize, city performance depends on the allocation of urban uses and activities; thus the results of this study focused on visual cognition is highly relevant to the everyday practice of urban planners and designers. Turning a city's main streets over to the car and completely withdrawing commercial uses to the secondary streets as has happened in planned, modern cities worldwide - goes against the grain of urban evolution.

Despite the biases and the limitations of the method, the patterns observed in this study offer an explanation for the unwritten rules of the city self-organizing process by linking pedestrian behaviour, visibility and urban activities. Therefore this study sets a framework for an evidence-based design tool to assist planners in the evaluation of urban projects in the rapidly urbanizing world. 
Acknowledgments The financial support of the Israel Science Foundation (Grant No. 59/17), and the Joan and Reginald Coleman-Cohen Fund is gratefully acknowledged.

The authors sincerely thank Dr. Michael Natapov for his valuable assistance with statistical analysis.

Open Access This article is distributed under the terms of the Creative Commons Attribution 4.0 International License (http://creativecommons.org/licenses/by/4.0/), which permits unrestricted use, distribution, and reproduction in any medium, provided you give appropriate credit to the original author(s) and the source, provide a link to the Creative Commons license, and indicate if changes were made.

\section{References}

Andersson C, Frenken K, Hellervik A (2006) A complex network approach to urban growth. Environ Plan A 38(10):1941-1964

Barabasi A-L, Albert R (1999) Emergence of scaling in random networks. Science 286:509-511

Barthelemy M (2011) Spatial networks. Phys Rep 499:1-101

Batty M (2003) The emergence of cities: complexity and urban dynamics. Population English Edition 44:0-16 Batty M (2013) In: ) (ed) The new science of cities. MIT Press, Cambrige

Bettencourt LMA, Lobo J, Helbing D, Kuhnert C, West GB (2007) Growth, innovation, scaling, and the pace of life in cities. Proc Natl Acad Sci U S A 104:7301-7306. https://doi.org/10.1073/ pnas.0610172104

Bishop ID (2002) Determination of thresholds of visual impact: the case of wind turbines. Environment and Planning B: Planning and Design 29(5):707-718

Broido AD, Clauset A (2018). Scale-free networks are rare, 26-28. Retrieved from http://arxiv. org/abs/1801.03400

Cardillo A, Scellato S, Latora V, Porta S (2006) Structural properties of planar graphs of urban street patterns. Phys Rev E 73:066107

Carvalho R, Penn A (2004) Scaling and universality in the micro-structure of urban space. Physica A 332: 539-547

Clauset A, Shalizi CR, Newman ME (2009) Power-law distributions in empirical data. SIAM Rev 51(4):661703

Ducruet C, Beauguitte L (2014) Spatial science and network science: review and outcomes of a complex relationship. Netw Spat Econ 14:297

Eichler D, Bar-Gera H, Blachman M (2013) Vortex-based zero-conflict Design of Urban Road Networks. Netw Spat Econ 13:229

Erdös P, Renyi AR (1959) On random graphs. Publicationes Mathematicae 6:290-297

Florida R (2004) Cities and the creative class. Routledge, New York

Franz G, Mallot HA, and Wiener JM (2005) Graph-based models of space in architecture and cognitive science- a comparative analysis. Proceedings of the 17th International Conference on Systems Research Informatics and Cybernetics InterSymp'2005 Architecture Engineering and Construction of Built Environments (pp. 30-38). International Institute for Advanced Studies in Systems Research and Cybernetics. Retrieved from http://eprints.bournemouth.ac.uk/13803/1/licence.txt

Gibson JJ (1950) The perception of the visual world. Houghton Mifflin, Boston

Golledge RG, Gärling T (2002) Spatial behavior in transportation modeling and planning. In: Goulias K (ed) Transportation engineering handbook: methods and applications, vol 46. CRC Press, Boca Raton, pp 037

Gret-Regamy A, Bishop ID, Bebi P (2007) Predicting the scenic beauty value of mapped landscape changes in a mountainous region through the use of GIS. Environment and Planning B: Planning and Design 34(1): 50-67

Hillier B, Hanson J (1984) The social logic of space. Cambridge University Press, Cambridge

Hölscher C, Meilinger T, Vrachliotis G, Brösamle M, Knauff M (2006) Up the down staircase: wayfinding strategies and multi-level buildings. J Environ Psychol 26(4):284-299

Huang A, Levinson D (2011) Why retails cluster: an agent model of location choice on supply chains. Environment and Planning B: Planning and Design 38(1):82-94

Humphries MD, Gurney K (2008) Network "small-world-ness": a quantitative method for determining canonical network equivalence. PLoS One 3(4). https://doi.org/10.1371/journal.pone.0002051 
Jacomy M, Bastian M, Heymann S (2009) Gephi: an open source software for exploring and manipulating networks. Proceedings of the Third International AAAI Conference on Weblogs and Social Media (ICWSM'09), in American Journal of Sociology 2009:361-362

Jiang B (2005) A structural perspective on visibility patterns with a topographic surface. Trans GIS 9(4):475488

Jiang B (2007) A topological pattern of urban street networks: universality and peculiarity. Physica A 384 : $647-655$

Jiang B, Claramunt C (2002) Integration of space syntax into GIS: new perspectives for urban morphology. Trans GIS 6(3):295-309

Jiang B, Duan Y, Lu F, Yang T, Zhao J (2014) Topological structure of urban street networks from the perspective of degree correlations. Environment and Planning B 41(5):813-828

Jones O, Marshall P, Boujenko N (2008) Creating more people-friendly urban streets through "link and place" street planning and design. IATSS Research 32(1):14-25

Krüger MTJ (1979) An approach to built-form connectivity at an urban scale: system description and its representation. Environment and Planning B: Planning and Design 6:67-88

Lang E, Schaffer PV (2001) A comment on the market value of a room with a view. Landsc Urban Plan 55: $113-120$

Loewenstein L (1963) The location of urban land uses. Land Econ 39(4):407-420. https://doi. org/10.2307/3144845

Natapov A, Fisher-Gewirtzman D (2016) Visibility of urban activities and pedestrian routes: an experiment in a virtual environment. Comput Environ Urban Syst 58:60-70

Natapov A, Czamanski D, Fisher-Gewirtzman D (2013) Can visibility predict location? Visibility graph of food and drink facilities in the city. Surv Rev 45(333):462-471(10)

Newman MEJ (2010) Networks: an introduction. Oxford University Press, Oxford

Oldenburg R (1989) Great good place; Cafes, Coffe shops, Bookstores, Bars, Hair Salons, and Other Hangouts at the Heart of a Community (Marlow \& Company)

Ottaviano G, I P, Puga D (1998) Agglomeration in the global economy: a survey of the new economic geography. World Econ 21:707-731

Porta S, Crucitti P, Latora V (2006) The network analysis of urban streets: a dual approach. Physica A 369:853

Sainte-Marieork M (2004) The Road to Direction Assessing the Impact of Road Asymmetry on Street Network Small-Worldness. In Spatial Cognition IX, Lecture Notes in Computer Science, pp. 206-221

Sevtsuk A, Mekonne M (2012) Urban network analysis. A new toolbox for ArcGIS. Revue Internationale de Géomatique 22(2):287-305

Shang H, Bishop ID (2000) Visual thresholds for detection, recognition and visual impact in landscape settings. J Environ Psychol 20(2):125-140

Strano E, Vincenzo N, Latora V, Porta S, and Barthélemy M (2012) Elementary processes governing the evolution of road networks. Scientific Reports 2:1-8. Retrieved March 1, 2012 (http://www.nature. com/doifinder/10.1038/srep00296

Wang L, Cohen AS, Carr M (2014) Spatial ability at two scales of representation: a meta-analysis. Learn Individ Differ 36:140-144

Watts DJ, Strogatz DH (1998) Collective dynamics of small-world networks. Nature 393:440

Weisman J (1981) Evaluating architectural legibility: way-finding in the built environment. Environ Behav 13: 189-204

Xie F, Levinson D (2009) Modeling the growth of transportation networks: a comprehensive review. Netw Spat Econ 9:291 